Article

\title{
Degrees of $L$-Continuity for Mappings between L-Topological Spaces
}

\author{
Zhenyu $\mathrm{Xiu}^{1}$ and Qinghua $\mathrm{Li}^{2, *}$ \\ 1 College of Applied Mathematics, Chengdu University of Information Technology, \\ Chengdu 610000, China; xzy@cuit.edu.cn \\ 2 School of Mathematics and Information Sciences, Yantai University, Yantai 264005, China \\ * Correspondence:lqh@ytu.edu.cn; Tel.: +86-13880310292
}

Received: 15 September 2019; Accepted: 17 October 2019; Published: 24 October 2019

\begin{abstract}
By means of the residual implication on a frame $L$, a degree approach to $L$-continuity and $L$-closedness for mappings between $L$-cotopological spaces are defined and their properties are investigated systematically. In addition, in the situation of $L$-topological spaces, degrees of $L$-continuity and of $L$-openness for mappings are proposed and their connections are studied. Moreover, if $L$ is a frame with an order-reversing involution', where $b^{\prime}=b \rightarrow \perp$ for $b \in L$, then degrees of $L$-continuity for mappings between $L$-cotopological spaces and degrees of $L$-continuity for mappings between $L$-topological spaces are equivalent.
\end{abstract}

Keywords: $L$-cotopological space; $L$-topological space; degree of $L$-continuity; degree of $L$-closedness; degree of $L$-openness

\section{Introduction}

Since Chang [1] introduced fuzzy set theory to topology, fuzzy topology and its related theories have been widely investigated such as [1-18]. The degree approach that equips fuzzy topology and its related structures with some degree description is also an essential character of fuzzy set theory. This approach has been developed extensively in the theory of fuzzy topology, fuzzy convergence and fuzzy convex structure. Yue and Fang [19] introduced a degree approach to $T_{1}$ and $T_{2}$ separation properties in $(L, M)$-fuzzy topological spaces. Shi $[20,21]$ defined the degrees of separation axioms which are compatible with $(L, M)$-fuzzy metric spaces. Li and Shi [22] introduced the degree of compactness in L-fuzzy topological spaces. Pang defined the compact degree of $(L, M)$-fuzzy convergence spaces [23] and degrees of $T_{i}(i=0,1,2)$ separation property as well as the regular property of stratified $L$-generalized convergence spaces [24]. All of the above-mentioned research mainly equipped spatial properties with some of the degree descriptions.

Actually, special mappings between structured spaces and the structured space itself can also be endowed with some degrees. Xiu and his co-authors $[25,26]$ defined degrees of fuzzy convex structures, fuzzy closure systems and fuzzy Alexandrov topologies and discussed their properties. Xiu and Pang [27] gave a degree approach to special mappings between $M$-fuzzifying convex spaces. In [28], Pang defined degrees of continuous mappings and open mappings between $L$-fuzzifying topological spaces to describe how a mapping between $L$-fuzzifying topological spaces becomes a continuous mapping or an open mapping in a degree sense. Liang and Shi [29] further defined the degrees of continuous mappings and open mappings between $L$-fuzzy 
topological spaces and investigated their relationship. Li [30] defined the degrees of special mappings in the theory of $L$-convex spaces and investigated their properties. Xiu and Pang [27] developed the degree approach to $M$-fuzzifying convex spaces to define the degrees of $M-C P$ mappings and $M-C C$ mappings.

Following this direction, we will focus on the case of $L$-cotopological spaces and $L$-topological spaces in this paper. By means of $L$-closure operators and $L$-interior operators, we will consider degrees of $L$-continuity and $L$-closedness for mappings between $L$-cotopological spaces as well as degrees of $L$-continuity and $L$-openness for mappings between $L$-topological spaces and will investigate their properties systematically.

\section{Preliminaries}

In this paper, let $L$ be a frame, $X$ be a nonempty set and $L^{X}$ be the set of all $L$-subsets on $X$. The bottom element and the top element of $L$ are denoted by $\perp$ and $T$, respectively. A residual implication can be defined by $a \rightarrow b=\bigvee\{\lambda \in L \mid a \wedge \lambda \leqslant b\}$. The operators on $L$ can be translated onto $L^{X}$ in a pointwise way. In this case, $L^{X}$ is also a complete lattice. Let $\perp$ and $I$ denote the smallest element and the largest element in $L^{X}$, respectively

Let $f: X \longrightarrow Y$ be a mapping. Define $f_{L}^{\rightarrow}: L^{X} \longrightarrow L^{Y}$ and $f_{L}^{\leftarrow}: L^{Y} \longrightarrow L^{X}$ by $f_{L} \rightarrow(B)(y)=$ $\bigvee_{f(x)=y} B(x)$ for $B \in L^{X}$ and $y \in Y$, and $f_{L}^{\leftarrow}(A)(x)=A(f(x))$ for $A \in L^{Y}$ and $x \in X$, respectively.

Using the residual implication, the concept of fuzzy inclusion order of $L$-subsets is introduced.

Definition 1 ([2,31]). The fuzzy inclusion order of L-subsets is a mapping $\mathbb{S}_{X}(-,-): L^{X} \times L^{X} \longrightarrow L$ which satisfying for each $C, D \in L^{X}$,

$$
\mathbb{S}_{X}(C, D)=\bigwedge_{x \in X}(C(x) \rightarrow D(x))
$$

Lemma 1 ([2,31]). Let $f: X \longrightarrow Y$ be a mapping. Then, for each $C, D, E \in L^{X}$ and $A, B \in L^{Y}$, the following statements hold:

(1) $\mathbb{S}_{X}(D, E)=T$ if and only if $D \leqslant E$.

(2) $D \leqslant E$ implies $\mathbb{S}_{X}(D, C) \geqslant \mathbb{S}_{X}(E, C)$.

(3) $D \leqslant$ E implies $\mathbb{S}_{X}(C, D) \leqslant \mathbb{S}_{X}(C, E)$.

(4) $\mathbb{S}_{X}(D, E) \wedge \mathbb{S}_{X}(E, C) \leqslant \mathbb{S}_{X}(D, C)$.

(5) $\mathbb{S}_{X}(D, E) \leqslant \mathbb{S}_{X}\left(f_{L} \rightarrow(D), f_{L} \rightarrow(E)\right)$.

(6) $\quad \mathbb{S}_{Y}(A, B) \leqslant \mathbb{S}_{Y}\left(f_{L}^{\leftarrow}(A), f_{L}^{\leftarrow}(B)\right)$

In the following, we will only use $\mathbb{S}$ to represent the fuzzy inclusion order of L-subsets on both $L^{X}$ and $L^{Y}$, not $\mathbb{S}_{X}$ or $\mathbb{S}_{Y}$. This will not lead to ambiguity in the paper.

Definition $2([1,18,32,33])$. Let $\mathcal{C}$ be a subset of $L^{X} . \mathcal{C}$ is called an L-cotopology on $X$ if it satisfies:

$(L C T 1) \perp, I \in \mathcal{C}$;

(LCT2) if $A, B \in \mathcal{C}$, then $A \vee B \in \mathcal{C}$;

(LCT3) if $\left\{A_{j} \mid j \in J\right\} \subseteq \mathcal{C}$, then $\bigwedge_{j \in J} A_{j} \in \mathcal{C}$.

For an $L$-cotopology $\mathcal{C}$ on $X$, the pair $(X, \mathcal{C})$ is called an $L$-cotopological space.

If $\mathcal{C}$ also satisfies:

(SLCT) for each $a \in L, \underline{a} \in \mathcal{C}$,

then it is called a stratified L-cotopology and the pair $(X, \mathcal{C})$ is called a stratified L-cotopological space. 
A mapping $f:\left(X, \mathcal{C}_{X}\right) \rightarrow\left(Y, \mathcal{C}_{Y}\right)$ is called L-continuous provided that, for each $B \in L^{Y}, B \in \mathcal{C}_{Y}$ implies $f_{L}^{\leftarrow}(B) \in \mathcal{C}_{X}$.

A mapping $f:\left(X, \mathcal{C}_{X}\right) \rightarrow\left(Y, \mathcal{C}_{Y}\right)$ is called L-closed provided that, for each $A \in L^{Y}, A \in \mathcal{C}_{X}$ implies $f_{L} \rightarrow(B) \in \mathcal{C}_{Y}$

Definition $3([18,32,33])$. An L-closure operator on $X$ is a mapping $C l: L^{X} \longrightarrow L^{X}$ which satisfies:

(LCL1) $\mathrm{Cl}(\perp)=\perp$;

(LCL2) $A \leqslant C l(A)$;

(LCL3) $\mathrm{Cl}(\mathrm{Cl}(A))=\mathrm{Cl}(A)$;

$(\mathrm{LCL} 4) \mathrm{Cl}(A \vee B)=\mathrm{Cl}(A) \vee \mathrm{Cl}(B)$.

For an L-closure operator $\mathrm{Cl}$ on $\mathrm{X}$, the pair $(\mathrm{X}, \mathrm{Cl})$ is called an L-closure space.

A mapping $f:\left(X, C l_{X}\right) \longrightarrow\left(Y, C l_{Y}\right)$ is called $L$-continuous provided that

$$
\forall A \in L^{X}, f_{L}\left(C l_{X}(A)\right) \leqslant C l_{Y}\left(f_{L}(A)\right) .
$$

It was proved in $[18,32,33]$ that $L$-cotopologies and $L$-closure operators are conceptually equivalent with transferring process $C l^{\mathcal{C}}(A)=\bigwedge\left\{B \in L^{X} \mid A \leqslant B \in \mathcal{C}\right\}$ for each $A \in L^{X}$ and $\mathcal{C}^{C l}=\left\{A \in L^{X} \mid C l(A)=A\right\}$. Correspondingly, $L$-continuous mappings between $L$-cotopological spaces and $L$-continuous mappings between $L$-closure spaces are compatible. In the sequel, we treat $L$-cotopological spaces with their $L$-continuous mappings and $L$-closure spaces with their $\mathrm{L}$-continuous mappings equivalently. We will use $\mathrm{Cl}$ to represent $\mathrm{Cl}^{\mathcal{C}}$ tacitly.

Definition 4 ([1,18,32,33]). An L-topology on $X$ is a subset $\mathcal{T} \subseteq L^{X}$ which satisfies:

(LT1) $\perp, I \in \mathcal{T}$;

(LT2) if $A, B \in \mathcal{T}$, then $A \wedge B \in \mathcal{T}$;

(LT3) if $\left\{A_{j} \mid j \in J\right\} \subseteq \mathcal{T}$, then $\bigvee_{j \in J} A_{j} \in \mathcal{T}$.

For an L-topology $\mathcal{T}$ on $X$, the pair $(X, \mathcal{T})$ is called an L-topological space.

A mapping $f:\left(X, \mathcal{T}_{X}\right) \rightarrow\left(Y, \mathcal{T}_{Y}\right)$ is called L-continuous provided that for each $B \in L^{Y}, B \in \mathcal{T}_{Y}$ implies $f_{L}^{\leftarrow}(B) \in \mathcal{T}_{X}$.

A mapping $f:\left(X, \mathcal{T}_{X}\right) \rightarrow\left(Y, \mathcal{T}_{Y}\right)$ is called L-open provided that for each $A \in L^{X}, A \in \mathcal{T}_{X}$ implies $f_{L} \rightarrow(B) \in \mathcal{T}_{Y}$

Definition $5([18,33])$. An L-interior operator on $X$ is a mapping $\mathcal{N}: L^{X} \longrightarrow L^{X}$ which satisfies:

$(\mathrm{LN} 1) \mathcal{N}(\underline{I})=\underline{\mathrm{I}}$

$(\mathrm{LN} 2) \mathcal{N}(C) \leqslant C ;$

$(\mathrm{LN} 3) \mathcal{N}(\mathcal{N}(C))=\mathcal{N}(C)$

$(\mathrm{LN} 4) \mathcal{N}(C \wedge D)=\mathcal{N}(C) \wedge \mathcal{N}(D)$.

For an $L$-interior operator $\mathcal{N}$ on $X$, the pair $(X, \mathcal{N})$ is called an L-interior space.

A mapping $f:\left(X, \mathcal{N}_{X}\right) \longrightarrow\left(Y, \mathcal{N}_{Y}\right)$ is called $L$-continuous provided that

$$
\forall A \in L^{X}, f_{L} \rightarrow\left(\mathcal{N}_{X}(A)\right) \leqslant \mathcal{N}_{Y}\left(f_{L}^{\rightarrow}(A)\right) .
$$

It was proved in $[18,33]$ that $L$-topologies and $L$-interior operators are conceptually equivalent with transferring process $\mathcal{N}^{\mathcal{T}}(A)=\bigvee\left\{B \in L^{X} \mid B \leqslant A, B \in \mathcal{T}\right\}$ for each $A \in L^{X}$ and $\mathcal{T}^{\mathcal{N}}=\left\{A \in L^{X} \mid \mathcal{N}(A)=A\right\}$. Correspondingly, $L$-continuous mappings between $L$-topological spaces and $L$-continuous mappings between $L$-interior spaces are compatible. In the sequel, we 
treat $L$-topological spaces with their $L$-continuous mappings and $L$-interior spaces with their $L$-continuous mappings equivalently. We will use $\mathcal{N}$ to represent $\mathcal{N}^{\mathcal{T}}$ tacitly.

Definition $6([3,32])$. An L-filter on $X$ is a mapping $\digamma: L^{X} \longrightarrow L$ which satisfies:

(F1) $\digamma(\perp)=\perp, \digamma(\underline{T})=T$;

$(F 2) \digamma(C \wedge D)=\digamma(C) \wedge \digamma(D)$ for each $C, D \in L^{X}$.

Let $\mathfrak{F}_{L}^{s}(X)$ denote the family of all L-filters on $X$.

Definition 7 ([3]). An L-fuzzy convergence on $X$ is a mapping $\operatorname{Lim}: \mathfrak{F}_{L}^{s}(X) \longrightarrow L^{X}$ which satisfies:

(L1) $\forall x \in X, \operatorname{Lim}[x](x)=\top$;

(L2) $\digamma_{1} \leqslant \digamma_{2}$ implies $\operatorname{Lim}\left(\digamma_{1}\right) \leqslant \operatorname{Lim}\left(\digamma_{2}\right)$.

For an $L$-fuzzy convergence $\operatorname{Lim}$ on $X$, the pair $(X, \mathrm{Lim})$ is called an $L$-fuzzy convergence space.

Theorem 1 ([3]). For an L-topological space $(X, \mathcal{T})$, let $\mathcal{N}$ be its interior operator and $\mathcal{U}^{x}$ be the $L$-neighborhood filter defined by $\mathcal{U}^{x}(A)=\mathcal{N}(A)(x)$ for each $x \in X$. Then, the mapping $\operatorname{Lim}: \mathfrak{F}_{L}^{s}(X) \longrightarrow$ $L^{X}$ defined by

$$
\operatorname{Lim}(\digamma)(x)=\bigwedge_{A \in L^{X}}\left(\mathcal{U}^{x}(A) \rightarrow \digamma(A)\right)
$$

is an L-fuzzy convergence on $X$.

\section{Degrees of $L$-Continuity and $L$-Closedness for Mappings between $L$-Cotopological Spaces}

In this section, we mainly define degrees of $L$-continuity of mappings and $L$-closedness of mappings to equip each mapping between $L$-cotopological spaces with some degree to be an $L$-continuous mapping and an $L$-closed mapping, respectively. Then, we will study their connections in a degree sense. Moreover, $f$ always denotes a mapping from $X$ to $Y$ and $g$ always denotes a mapping from $Y$ to $Z$ in the following sections.

Definition 8. Let $\left(X, \mathcal{C}_{X}\right)$ and $\left(Y, \mathcal{C}_{Y}\right)$ be L-cotopological spaces. Then,

(1) $D_{c_{1}}(f)$ defined by

$$
D_{c_{1}}(f)=\bigwedge_{B \in L^{X}} \mathbb{S}\left(f_{L} \rightarrow\left(C l_{X}(B)\right), C l_{Y}\left(f_{L} \rightarrow(B)\right)\right)
$$

is called the degree of L-continuity for $f$.

(2) $D_{b}(f)$ defined by

$$
D_{b}(f)=\bigwedge_{A \in L^{X}} \mathbb{S}\left(C l_{Y}\left(f_{L}(A)\right), f_{L}\left(C l_{X}(A)\right)\right)
$$

is called the degree of L-closedness for $f$.

Remark 1. (1) If $D_{c_{1}}(f)=T$, then $f_{L}\left(C l_{X}(A)\right) \leqslant C l_{Y}\left(f_{L}(A)\right)$ for all $A \in L^{X}$, which is exactly the definition of $L$-continuous mappings between $L$-closure spaces. As we claimed that $L$-continuous mappings between $L$-cotopological spaces and L-continuous mappings between $L$-closure spaces are compatible, we don't distinguish them. Therefore, we defined the degree of L-continuity of mappings between L-cotopological spaces by using L-continuous mappings between their induced L-closure spaces.

(2) If $D_{b}(f)=T$, then $C l_{Y}\left(f_{L} \rightarrow(A)\right) \leqslant f_{L}\left(C l_{X}(A)\right)$ for all $A \in L^{X}$. This is exactly the equivalent form of L-closed mappings between $L$-cotopological spaces by means of the corresponding $L$-closure operators. 
Lemma 2. Let $f: X \longrightarrow Y$ and $g: Y \longrightarrow Z$ be mappings. Then, for each $A \in L^{X}, B \in L^{Y}$ and $C \in L^{Z}$, the following statements hold:

(1) $\quad A \leqslant f_{L}^{\leftarrow}\left(f_{L} \rightarrow(A)\right)$. If $f$ is injective, then the equality holds.

(2) $f_{L} \rightarrow\left(f_{L}^{\leftarrow}(B)\right) \leqslant B$. If $f$ is surjective, then the equality holds.

(3) $(g \circ f)_{\vec{L}}(A)=g_{\vec{L}}\left(f_{L}(A)\right)$.

(4) $\quad(g \circ f)_{L}^{\overleftarrow{L}}(C)=f_{L}^{\leftarrow}\left(g_{L}^{\leftarrow}(C)\right)$

Proof. The proofs are routine and are omitted.

Theorem 2. Let $\left(X, \mathcal{C}_{X}\right)$ and $\left(Y, \mathcal{C}_{Y}\right)$ be L-cotopological spaces. Then,

$$
D_{c_{1}}(f)=\bigwedge_{B \in L^{Y}} \mathbb{S}\left(f_{L} \rightarrow\left(C l_{X}\left(f_{L}^{\leftarrow}(B)\right)\right), C l_{Y}(B)\right)
$$

Proof. It follows from the definition of $D_{c_{1}}(f)$ that

$$
\begin{aligned}
D_{c_{1}}(f) & =\bigwedge_{A \in L^{X}} \mathbb{S}\left(f_{L}\left(C l_{X}(A)\right), C l_{Y}\left(f_{L}(A)\right)\right) \\
& \leqslant \bigwedge_{B \in L^{Y}} \mathbb{S}\left(f_{L}^{\rightarrow}\left(C l_{X}\left(f_{L}^{\leftarrow}(B)\right)\right), C l_{Y}\left(f_{L} \rightarrow\left(f_{L}^{\leftarrow}(B)\right)\right)\right) \\
& \leqslant \bigwedge_{B \in L^{Y}} \mathbb{S}\left(f_{L}^{\rightarrow}\left(C l_{X}\left(f_{L}^{\leftarrow}(B)\right)\right), C l_{Y}(B)\right) \\
& \left.\leqslant \bigwedge_{A \in L^{X}} \mathbb{S}\left(f_{L} \rightarrow C l_{X}\left(f_{L}^{\leftarrow}\left(f_{L} \rightarrow(A)\right)\right)\right), C l_{Y}\left(f_{L}(A)\right)\right) \\
& \leqslant \bigwedge_{A \in L^{X}} \mathbb{S}\left(f_{L}\left(C l_{X}(A)\right), C l_{Y}\left(f_{L} \rightarrow(A)\right)\right) \\
& =D_{c_{1}}(f) .
\end{aligned}
$$

This implies

$$
D_{c_{1}}(f)=\bigwedge_{B \in L^{Y}} \mathbb{S}\left(f_{L} \rightarrow\left(C l_{X}\left(f^{\leftarrow}(B)\right)\right), C l_{Y}(B)\right)
$$

as desired.

Theorem 3. (1) If id : $\left(X, \mathcal{C}_{X}\right) \longrightarrow\left(X, \mathcal{C}_{X}\right)$ is the identity mapping, then $D_{\mathcal{c}_{1}}($ id $)=\top$ and $D_{b}(i d)=\top$. (2) If $y_{0}:\left(X, \mathcal{C}_{X}\right) \longrightarrow\left(Y, \mathcal{C}_{Y}\right)$ is a constant mapping between stratified L-cotopological spaces with the constant $y_{0} \in Y$, then $D_{c_{1}}\left(y_{0}\right)=T$.

Proof. (1) Straightforward,

(2) It follows immediately from the definition of $D_{c_{1}}\left(y_{0}\right)$ that

$$
\begin{aligned}
D_{c_{1}}\left(y_{0}\right) & =\bigwedge_{A \in L^{X}} \mathbb{S}\left(C l_{X}(A),\left(y_{0}\right)_{L}^{\overleftarrow{L}}\left(C l_{Y}\left(\left(y_{0}\right)_{L}(A)\right)\right)\right) \\
& =\bigwedge_{A \in L^{X}} \bigwedge_{x \in X}\left(C l_{X}(A)(x) \rightarrow C l_{Y}\left(\left(y_{0}\right)_{L}(A)\right)\left(y_{0}\right)\right)
\end{aligned}
$$


Since $\left(X, \mathcal{C}_{X}\right)$ is stratified, we know $\bigvee_{z \in X} A(z) \in \mathcal{C}_{X}$. Then, for each $A \in L^{X}$ and $x \in X$, it follows that

$$
C l_{X}(A)(x)=\bigwedge\left\{B \in L^{X} \mid A \leqslant B \in \mathcal{C}_{X}\right\} \leqslant \bigvee_{z \in X} A(z) .
$$

Furthermore, we have

$$
C l_{Y}\left(\left(y_{0}\right)_{L}(A)\right)\left(y_{0}\right) \geqslant\left(y_{0}\right)_{L}(A)\left(y_{0}\right)=\bigvee_{y_{0}(z)=y_{0}} A(z)=\bigvee_{z \in X} A(z) \geqslant C l_{X}(A)(x)
$$

This implies that $C l_{X}(A)(x) \leqslant C l_{Y}\left(\left(y_{0}\right)_{L}(A)\right)\left(y_{0}\right)$ for each $A \in L^{X}$ and $x \in X$. Therefore, we have $D_{c_{1}}\left(y_{0}\right)=\top$.

Next, we give another characterizations of degrees of $L$-continuty for mappings between $L$-cotopological spaces.

Theorem 4. Let $\left(X, \mathcal{C}_{X}\right)$ and $\left(Y, \mathcal{C}_{Y}\right)$ be L-cotopological spaces. Then,

$$
D_{c_{1}}(f)=\bigwedge_{A \in L^{X}} \mathbb{S}\left(C l_{X}(A), f_{L}^{\leftarrow}\left(C l_{Y}\left(f_{L}(A)\right)\right)\right)
$$

Proof. By the definition of $\mathbb{S}$, it follows that

$$
\begin{aligned}
& \mathbb{S}\left(f_{L}\left(C l_{X}(A)\right), C l_{Y}\left(f_{L}^{\rightarrow}(A)\right)\right) \\
= & \bigwedge_{y \in Y}\left(f_{L}^{\rightarrow}\left(C l_{X}(A)\right)(y) \rightarrow C l_{Y}\left(f_{L}^{\rightarrow}(A)\right)(y)\right) \\
= & \bigwedge_{y \in Y}\left(\bigvee_{f(x)=y} C l_{X}(A)(x) \rightarrow C l_{Y}\left(f_{L} \rightarrow(A)\right)(y)\right) \\
= & \bigwedge_{y \in Y} \bigwedge_{f(x)=y}\left(C l_{X}(A)(x) \rightarrow C l_{Y}\left(f_{L}^{\rightarrow}(A)\right)(f(x))\right) \\
= & \bigwedge_{y \in Y} \bigwedge_{f(x)=y}\left(C l_{X}(A)(x) \rightarrow f_{L}^{\leftarrow}\left(C l_{Y}\left(f_{L}^{\rightarrow}(A)\right)\right)(x)\right) \\
= & \bigwedge_{x \in X}\left(C l_{X}(A)(x) \rightarrow f_{L}^{\leftarrow}\left(C l_{Y}\left(f_{L}^{\rightarrow}(A)\right)\right)(x)\right) \\
= & \mathbb{S}\left(C l_{X}(A), f_{L}^{\leftarrow}\left(C l_{Y}\left(f_{L}^{\rightarrow}(A)\right)\right)\right) .
\end{aligned}
$$

This means

$$
\begin{aligned}
D_{c_{1}}(f) & =\bigwedge_{A \in L^{X}} \mathbb{S}\left(f_{L} \rightarrow\left(C l_{X}(A)\right), C l_{Y}\left(f_{L}(A)\right)\right) \\
& =\bigwedge_{A \in L^{X}} \mathbb{S}\left(C l_{X}(A), f_{L}^{\leftarrow}\left(C l_{Y}\left(f_{L}^{\rightarrow}(A)\right)\right)\right),
\end{aligned}
$$

as desired. 
Theorem 5. Let $\left(X, \mathcal{C}_{X}\right)$ and $\left(Y, \mathcal{C}_{Y}\right)$ be L-cotopological spaces. Then,

$$
D_{c_{1}}(f)=\bigwedge_{B \in L^{Y}} \mathbb{S}\left(C l_{X}\left(f_{L}^{\leftarrow}(B)\right), f_{L}^{\leftarrow}\left(C l_{Y}(B)\right)\right)
$$

Proof.

$$
\begin{aligned}
& \bigwedge_{B \in L^{Y}} \mathbb{S}\left(C l_{X}\left(f_{L}^{\leftarrow}(B)\right), f_{L}^{\leftarrow}\left(C l_{Y}(B)\right)\right) \\
\leqslant & \bigwedge_{A \in L^{X}} \mathbb{S}\left(C l_{X}\left(f_{L}^{\leftarrow}\left(f_{L}^{\rightarrow}(A)\right)\right), f_{L}^{\leftarrow}\left(C l_{Y}\left(f_{L}^{\rightarrow}(A)\right)\right)\right) \\
\leqslant & \bigwedge_{A \in L^{X}} \mathbb{S}\left(C l_{X}(A), f_{L}^{\leftarrow}\left(C l_{Y}\left(f_{L}^{\rightarrow}(A)\right)\right)\right) \\
= & D_{c_{1}}(f) .
\end{aligned}
$$

By the definition of $\mathbb{S}$, it follows that

$$
\begin{aligned}
& \mathbb{S}\left(C l_{X}\left(f_{L}^{\leftarrow}(B)\right), f_{L}^{\leftarrow}\left(C l_{Y}(B)\right)\right) \\
= & \bigwedge_{x \in X}\left(C l_{X}\left(f_{L}^{\leftarrow}(B)\right)(x) \rightarrow f_{L}^{\leftarrow}\left(C l_{Y}(B)\right)(x)\right) \\
= & \bigwedge_{x \in X}\left(C l_{X}\left(f_{L}^{\leftarrow}(B)\right)(x) \rightarrow\left(C l_{Y}(B)\right)(f(x))\right) \\
\geqslant & \bigwedge_{y \in Y}\left(C l_{X}\left(f_{L}^{\leftarrow}(B)\right)\left(f^{-1}(y)\right) \rightarrow\left(C l_{Y}(B)\right)(y)\right) \\
\geqslant & \bigwedge_{y \in Y}\left(\bigvee_{f(x)=y} C l_{X}\left(f_{L}^{\leftarrow}(B)\right)(x) \rightarrow\left(C l_{Y}(B)\right)(y)\right) \\
= & \bigwedge_{y \in Y}\left(f_{L}^{\rightarrow}\left(C l_{X}\left(f_{L}^{\leftarrow}(B)\right)\right)(y) \rightarrow\left(C l_{Y}(B)\right)(y)\right) \\
= & D_{c_{1}}(f) .
\end{aligned}
$$

This implies

$$
D_{c_{1}}(f)=\bigwedge_{B \in L^{Y}} \mathbb{S}\left(C l_{X}\left(f_{L}^{\leftarrow}(B)\right), f_{L}^{\leftarrow}\left(C l_{Y}(B)\right)\right)
$$

as desired.

In $L$-cotopological spaces, compositions of $L$-continuous mappings (resp. $L$-closed mappings) are still $L$-continuous mappings (resp. L-closed mappings). Now, let us give a degree representation of this result.

Theorem 6. For L-cotopological spaces $\left(X, \mathcal{C}_{X}\right),\left(Y, \mathcal{C}_{Y}\right)$ and $\left(Z, \mathcal{C}_{Z}\right)$, the following inequalities hold:

(1) $D_{\mathcal{C}_{1}}(f) \wedge D_{\mathcal{C}_{1}}(g) \leqslant D_{\mathcal{C}_{1}}(g \circ f)$.

(2) $D_{b}(f) \wedge D_{b}(g) \leqslant D_{b}(g \circ f)$. 
Proof. (1) By Theorem 4, we have

$$
\begin{aligned}
& D_{\mathcal{C}_{1}}(f) \wedge D_{\mathcal{C}_{1}}(g) \\
& =\bigwedge_{A \in L^{X}} \mathbb{S}\left(f_{L} \rightarrow\left(C l_{X}(A)\right), C l_{Y}\left(f_{L} \rightarrow(A)\right)\right) \wedge \bigwedge_{B \in L^{Y}} \mathbb{S}\left(g_{L} \vec{L}\left(C l_{Y}(B)\right), C l_{Z}\left(g_{\vec{L}}(B)\right)\right) \\
& \leqslant \bigwedge_{A \in L^{X}} \mathbb{S}\left(f_{L}^{\rightarrow}\left(C l_{X}(A)\right), C l_{Y}\left(f_{L} \rightarrow(A)\right)\right) \wedge \bigwedge_{B \in L^{Y}} \mathbb{S}\left(C l_{Y}(B), g_{L}^{\leftarrow}\left(C l_{Z}\left(g_{L} \vec{L}(B)\right)\right)\right) \\
& \leqslant \bigwedge_{A \in L^{X}}\left(\mathbb{S}\left(f_{L} \rightarrow\left(C l_{X}(A)\right), C l_{Y}\left(f_{L} \rightarrow(A)\right)\right) \wedge \mathbb{S}\left(C l_{Y}\left(f_{L}(A)\right), g_{L}^{\overleftarrow{L}}\left(C l_{Z}\left(g_{L}\left(f_{L}(A)\right)\right)\right)\right)\right) \\
& \leqslant \bigwedge_{A \in L^{X}} \mathbb{S}\left(f_{L} \vec{C}\left(C l_{X}(A)\right), g_{L}^{\leftarrow}\left(C l_{Z}\left((g \circ f)_{L}(A)\right)\right)\right) \\
& =\bigwedge_{A \in L^{X}} \mathbb{S}\left((g \circ f)_{L}\left(C l_{X}(A)\right), C l_{Z}\left((g \circ f)_{L}(A)\right)\right) \\
& =D_{c_{1}}(g \circ f) \text {. }
\end{aligned}
$$

(2) Adopting the proof of (1), it can be verified directly.

Next, we investigate the connections between degrees of L-continuity and that of L-closedness.

Theorem 7. For L-cotopological spaces $\left(X, \mathcal{C}_{X}\right),\left(Y, \mathcal{C}_{Y}\right)$ and $\left(Z, \mathcal{C}_{Z}\right)$, if $g$ is injective, then the following inequality holds:

$$
D_{b}(g \circ f) \wedge D_{c_{1}}(g) \leqslant D_{b}(f) .
$$

Proof. If $g$ is an injective mapping, then we have $g_{L}^{\overleftarrow{L}}\left(g_{L}(B)\right)=B$ for all $B \in L^{Y}$. Then, it follows that

$$
\begin{aligned}
& D_{b}(g \circ f) \wedge D_{c_{1}}(g)=\bigwedge_{A \in L^{X}} \mathbb{S}\left(C l_{Z}\left((g \circ f)_{L}(A)\right),(g \circ f)_{L}\left(C l_{X}(A)\right)\right) \\
& \wedge \bigwedge_{B \in L^{Y}} \mathbb{S}\left(g_{L} \vec{L}\left(C l_{Y}(B)\right), C l_{Z}\left(g_{L}(B)\right)\right) \\
& \leqslant \bigwedge_{A \in L^{X}} \mathbb{S}\left(C l_{Z}\left((g \circ f)_{L}(A)\right),(g \circ f)_{L}\left(C l_{X}(A)\right)\right) \\
& \wedge \bigwedge_{A \in L^{X}} \mathbb{S}\left(g_{L} \vec{C}\left(C_{Y}\left(f_{L}(A)\right)\right), C l_{Z}\left(g_{L} \vec{L}\left(f_{L}(A)\right)\right)\right) \\
& \leqslant \bigwedge_{A \in L^{X}} \mathbb{S}\left(C l_{Z}\left((g \circ f)_{L}(A)\right),(g \circ f)_{\vec{L}}\left(C l_{X}(A)\right)\right) \\
& \wedge \bigwedge_{A \in L^{X}} \mathbb{S}\left(g_{L} \vec{C}\left(C_{Y}\left(f_{L}(A)\right)\right), C l_{Z}\left((g \circ f)_{L}(A)\right)\right) \\
& \leqslant \bigwedge_{A \in L^{X}} \mathbb{S}\left(g_{\vec{L}}\left(C l_{Y}\left(f_{L}(A)\right)\right),(g \circ f)_{L}\left(C l_{X}(A)\right)\right) \\
& =\bigwedge_{A \in L^{X}} \mathbb{S}\left(C l_{Y}\left(f_{L} \overrightarrow{(A)}\right), g_{L}^{\leftarrow}\left((g \circ f)_{L}\left(C l_{X}(A)\right)\right)\right) \\
& =\bigwedge_{A \in L^{X}} \mathbb{S}\left(C l_{Y}\left(f_{L}(A)\right), f_{L}\left(C l_{X}(A)\right)\right) \\
& =D_{b}(f) \text {. }
\end{aligned}
$$


Theorem 8. For L-cotopological spaces $\left(X, \mathcal{C}_{X}\right),\left(Y, \mathcal{C}_{Y}\right)$ and $\left(Z, \mathcal{C}_{Z}\right)$, if $g$ is surjective, then the following inequality holds:

$$
D_{b}(g \circ f) \wedge D_{c_{1}}(f) \leqslant D_{b}(g) .
$$

Proof. If $f$ is a surjective mapping, then $f_{L} \rightarrow\left(f_{L}^{\leftarrow}(A)\right)=A$ for all $A \in L^{Y}$. Then, it follows that

$$
\begin{aligned}
& \bigwedge_{B \in L^{Y}} \mathbb{S}\left(C l_{Z}\left(g_{L}(B)\right), g_{L} \vec{L}\left(C l_{Y}(B)\right)\right) \\
= & \bigwedge_{B \in L^{Y}} \mathbb{S}\left(C l_{Z}\left(g_{L} \vec{L}\left(f_{L} \vec{f}\left(f_{L}^{\leftarrow}(B)\right)\right)\right), g_{L}\left(C l_{Y}\left(f_{L}\left(f_{L}^{\leftarrow}(B)\right)\right)\right)\right) \\
\geqslant & \left.\bigwedge_{A \in L^{X}} \mathbb{S}\left(C l_{Z}((g \circ f))_{L}(A)\right), g_{L}\left(C l_{Y}\left(f_{L}(A)\right)\right)\right) \\
\geqslant & \bigwedge_{B \in L^{Y}} \mathbb{S}\left(C l_{Z}\left(g_{L}(B)\right), g_{L}\left(C l_{Y}(B)\right)\right) .
\end{aligned}
$$

This shows

$$
\bigwedge_{B \in L^{Y}} \mathbb{S}\left(C l_{Z}\left(g_{L}(B)\right), g_{L}\left(C l_{Y}(B)\right)\right)=\bigwedge_{A \in L^{X}} \mathbb{S}\left(C l_{Z}\left((g \circ f)_{L}(A)\right), g_{L}\left(C l_{Y}\left(f_{L}(A)\right)\right)\right) .
$$

Then, we have

$$
\begin{aligned}
& D_{b}(g \circ f) \wedge D_{c_{1}}(f) \\
= & \bigwedge_{A \in L^{X}} \mathbb{S}\left(C l_{Z}\left((g \circ f)_{L}(A)\right),(g \circ f)_{L}\left(C l_{X}(A)\right)\right) \\
& \wedge \bigwedge_{A \in L^{X}} \mathbb{S}\left(f_{L} \rightarrow\left(C l_{X}(A)\right), C l_{Y}\left(f_{L}(A)\right)\right) \\
\leqslant & \bigwedge_{A \in L^{X}} \mathbb{S}\left(C l_{Z}\left((g \circ f)_{L}(A)\right),(g \circ f)_{L}\left(C l_{X}(A)\right)\right) \\
& \wedge \bigwedge_{A \in L^{X}} \mathbb{S}\left((g \circ f)_{L}\left(C l_{X}(A)\right), g_{L} \vec{L}\left(C l_{Y}\left(f_{L} \rightarrow(A)\right)\right)\right) \\
\leqslant & \bigwedge_{A \in L^{X}} \mathbb{S}\left(C l_{Z}\left((g \circ f)_{L}(A)\right), g_{L}\left(C l_{Y}\left(f_{L}(A)\right)\right)\right) \\
= & \bigwedge_{B \in L^{Y}} \mathbb{S}\left(C l_{Z}(g \rightarrow(B)), g_{L}\left(C l_{Y}(B)\right)\right) \\
= & D_{b}(g) .
\end{aligned}
$$

\section{Degrees of $L$-Continuity and $L$-Openness for Mappings between $L$-Topological Spaces}

In this section, we mainly define degrees of $L$-continuity and $L$-openness to equip each mapping between $L$-topological spaces with some degree to be an $L$-continuous mapping and an $L$-open mapping, respectively. Then, we will study their connections in a degree sense.

Definition 9. Let $\left(X, \mathcal{T}_{X}\right)$ and $\left(X, \mathcal{T}_{Y}\right)$ be L-topological spaces. 
(1) $D_{c_{2}}(f)$ defined by

$$
D_{\mathcal{C}_{2}}(f)=\bigwedge_{B \in L^{Y}} \mathbb{S}\left(f_{L}^{\leftarrow}\left(\mathcal{N}_{Y}(B)\right), \mathcal{N}_{X}\left(f_{L}^{\leftarrow}(B)\right)\right)
$$

is called the degree of L-continuity for $f$.

(2) $D_{k}(f)$ defined by

$$
D_{k}(f)=\bigwedge_{A \in L^{X}} \mathbb{S}\left(f_{L} \rightarrow\left(\mathcal{N}_{X}(A)\right), \mathcal{N}_{Y}\left(f_{L}(A)\right)\right)
$$

is called the degree of L-openness for $f$.

Remark 2. (1) If $D_{c_{2}}(f)=T$, then $f_{L}^{\leftarrow}\left(\mathcal{N}_{X}(B)\right) \leqslant \mathcal{N}_{Y}\left(f_{L}^{\leftarrow}(B)\right)$ for all $B \in L^{Y}$, which is exactly the definition of $L$-continuous mappings between $L$-interior spaces.

(2) If $D_{k}(f)=T$, then $f_{L} \rightarrow\left(\mathcal{N}_{X}(A)\right) \leqslant \mathcal{N}_{Y}\left(f_{L}(A)\right)$ for all $A \in L^{X}$. This is exactly the equivalent form of $L$-open mappings between $L$-topological spaces by means of the corresponding L-interior operators.

Theorem 9. Let $\left(X, \mathcal{T}_{X}\right)$ and $\left(X, \mathcal{T}_{Y}\right)$ be L-topological spaces. Then,

$$
D_{\mathcal{C}_{2}}(f)=\bigwedge_{x \in X} \bigwedge_{C \in L^{Y}}\left(\mathcal{U}_{Y}^{f(x)}(C) \rightarrow f\left(\mathcal{U}_{X}^{x}\right)(C)\right)
$$

Proof.

$$
\begin{aligned}
& \bigwedge_{x \in X} \bigwedge_{C \in L^{Y}}\left(\mathcal{U}_{Y}^{f(x)}(C) \rightarrow f\left(\mathcal{U}_{X}^{x}\right)(C)\right) \\
= & \bigwedge_{x \in X} \bigwedge_{C \in L^{Y}}\left(\mathcal{U}_{Y}^{f(x)}(C) \rightarrow\left(\mathcal{U}_{X}^{x}\right)\left(f_{L}^{\leftarrow}(C)\right)\right) \\
= & \bigwedge_{x \in X} \bigwedge_{C \in L^{Y}}\left(\mathcal{N}_{Y}(C)(f(x)) \rightarrow \mathcal{N}_{X}\left(f_{L}^{\leftarrow}(C)\right)(x)\right) \\
= & \bigwedge_{x \in X} \bigwedge_{C \in L^{Y}}\left(f_{L}^{\leftarrow}\left(\mathcal{N}_{Y}(C)\right)(x) \rightarrow \mathcal{N}_{X}\left(f_{L}^{\leftarrow}(C)\right)(x)\right) \\
= & \bigwedge_{C \in L^{Y}} \bigwedge_{x \in X}\left(f_{L}^{\leftarrow}\left(\mathcal{N}_{Y}(C)\right)(x) \rightarrow \mathcal{N}_{X}\left(f_{L}^{\leftarrow}(C)\right)(x)\right) \\
= & \bigwedge_{C \in L^{Y}} \mathbb{S}\left(f_{L}^{\leftarrow}\left(\mathcal{N}_{Y}(C)\right), \mathcal{N}_{X}\left(f_{L}^{\leftarrow}(C)\right)\right) \\
= & D_{c_{2}}(f) .
\end{aligned}
$$

Theorem 10. Let $\left(X, \mathcal{T}_{X}\right)$ and $\left(X, \mathcal{T}_{Y}\right)$ be L-topological spaces. Then,

$$
D_{\mathcal{C}_{2}}(f)=\bigwedge_{\digamma \in \mathcal{F}_{L}^{S}(X)} \mathbb{S}\left(\operatorname{Lim}_{X} \digamma, f_{L}^{\leftarrow}\left(\operatorname{Lim}_{Y} f(\digamma)\right)\right)
$$


Proof. On one hand,

$$
\begin{aligned}
& \bigwedge_{\digamma \in \mathfrak{F}_{L}^{S}(X)} \mathbb{S}\left(\operatorname{Lim}_{X} \digamma, f_{L}^{\leftarrow}\left(\operatorname{Lim}_{Y} f(\digamma)\right)\right) \\
= & \bigwedge_{\digamma \in \mathfrak{F}_{L}^{S}(X)} \bigwedge_{x \in X}\left(\operatorname{Lim}_{X} \digamma(x) \rightarrow f_{L}^{\leftarrow}\left(\operatorname{Lim}_{Y} f(\digamma)\right)(x)\right) \\
= & \bigwedge_{\digamma \in \mathfrak{F}_{L}^{S}(X)} \bigwedge_{x \in X}\left(\operatorname{Lim}_{X} \digamma(x) \rightarrow \operatorname{Lim}_{Y} f(\digamma)(f(x))\right) \\
= & \bigwedge_{\digamma \in \mathfrak{F}_{L}^{S}(X)} \bigwedge_{x \in X}\left(\bigwedge_{A \in L^{X}}\left(\mathcal{U}_{X}^{x}(A) \rightarrow \digamma(A)\right) \rightarrow \bigwedge_{B \in L^{Y}}\left(\mathcal{U}_{Y}^{f(x)}(B) \rightarrow f(\digamma)(B)\right)\right) \\
\geqslant & \bigwedge_{\digamma \in \mathfrak{F}_{L}^{S}(X)} \bigwedge_{x \in X}\left(\bigwedge_{B \in L^{Y}}\left(\mathcal{U}_{X}^{x}\left(f_{L}^{\leftarrow}(B)\right) \rightarrow \digamma\left(f_{L}^{\leftarrow}(B)\right)\right) \rightarrow \bigwedge_{B \in L^{Y}}\left(\mathcal{U}_{Y}^{f(x)}(B) \rightarrow f(\digamma)(B)\right)\right) \\
= & \bigwedge_{\digamma \in \mathfrak{F}_{L}^{S}(X)} \bigwedge_{x \in X}\left(\bigwedge_{B \in L^{Y}}\left(\mathcal{U}_{X}^{x}\left(f_{L}^{\leftarrow}(B)\right) \rightarrow f(\digamma)(B)\right) \rightarrow \bigwedge_{B \in L^{Y}}\left(\mathcal{U}_{Y}^{f(x)}(B) \rightarrow f(\digamma)(B)\right)\right) \\
\geqslant & \bigwedge_{x \in X} \bigwedge_{B \in L^{Y}}\left(\mathcal{U}_{Y}^{f(x)}(B) \rightarrow \mathcal{U}_{X}^{x}\left(f_{L}^{\leftarrow}(B)\right)\right) \\
= & \bigwedge_{x \in X} \bigwedge_{B \in L^{Y}}\left(\mathcal{U}_{Y}^{f(x)}(B) \rightarrow f\left(\mathcal{U}_{X}^{x}\right)(B)\right) \\
= & D_{\mathcal{C}_{2}}(f)
\end{aligned}
$$

On the other hand,

$$
\begin{aligned}
& \bigwedge_{\digamma \in \mathfrak{F}_{L}^{S}(X)} \mathbb{S}\left(\operatorname{Lim}_{X} \digamma, f_{L}^{\leftarrow}\left(\operatorname{Lim}_{Y} f(F)\right)\right) \\
= & \bigwedge_{\digamma \in \mathcal{F}_{L}^{S}(X)} \bigwedge_{x \in X}\left(\operatorname{Lim}_{X} \digamma(x) \rightarrow f_{L}^{\leftarrow}\left(\operatorname{Lim}_{Y} f(\digamma)\right)(x)\right) \\
= & \bigwedge_{\digamma \in \mathfrak{F}_{L}^{S}(X)} \bigwedge_{x \in X}\left(\operatorname{Lim}_{X} \digamma(x) \rightarrow \operatorname{Lim}_{Y} f(\digamma)(f(x))\right) \\
= & \bigwedge_{\digamma \in \mathfrak{F}_{L}^{S}(X)} \bigwedge_{x \in X}\left(\bigwedge_{A \in L^{X}}\left(\mathcal{U}_{X}^{x}(A) \rightarrow \digamma(A)\right) \rightarrow \bigwedge_{B \in L^{Y}}\left(\mathcal{U}_{Y}^{f(x)}(B) \rightarrow f(\digamma)(B)\right)\right) \\
\leqslant & \bigwedge_{x \in X}\left(\bigwedge_{A \in L^{X}}\left(\mathcal{U}_{X}^{x}(A) \rightarrow \mathcal{U}_{X}^{x}(A)\right) \rightarrow \bigwedge_{B \in L^{Y}}\left(\mathcal{U}_{Y}^{f(x)}(B) \rightarrow f\left(\mathcal{U}_{X}^{x}\right)(B)\right)\right) \\
= & \bigwedge_{x \in X} \bigwedge_{B \in L^{Y}}\left(\mathcal{U}_{Y}^{f(x)}(B) \rightarrow f\left(\mathcal{U}_{X}^{x}\right)(B)\right) \\
= & D_{\mathcal{C}_{2}}(f) .
\end{aligned}
$$

Therefore, $D_{\mathcal{C}_{2}}(f)=\bigwedge_{x \in X} \bigwedge_{B \in L^{Y}}\left(\mathcal{U}_{Y}^{f(x)}(B) \rightarrow f\left(\mathcal{U}_{X}^{x}\right)(B)\right)$.

In $L$-topological spaces, compositions of $L$-continuous mappings (resp. $L$-open mappings) are still $L$-continuous mappings (resp. $L$-open mappings). Now, let us give a degree representation of this result.

Theorem 11. For L-topological spaces $\left(X, \mathcal{T}_{X}\right),\left(Y, \mathcal{T}_{Y}\right)$ and $\left(Z, \mathcal{T}_{Z}\right)$, the following inequalities hold: (1) $D_{c_{2}}(f) \wedge D_{c_{2}}(g) \leqslant D_{c_{2}}(g \circ f)$. 
(2) $D_{k}(f) \wedge D_{k}(g) \leqslant D_{k}(g \circ f)$.

Proof. (1) By Definition 9, we have

$$
\begin{aligned}
& D_{c_{2}}(f) \wedge D_{c_{2}}(g) \\
& =\bigwedge_{A \in L^{Y}} \mathbb{S}\left(f_{L}^{\leftarrow}\left(\mathcal{N}_{Y}(A)\right), \mathcal{N}_{X}\left(f_{L}^{\leftarrow}(A)\right)\right) \\
& \wedge \bigwedge_{B \in L^{Z}} \mathbb{S}\left(g_{L}^{\leftarrow}\left(\mathcal{N}_{Z}(B)\right), \mathcal{N}_{Y}\left(g_{L}^{\leftarrow}(B)\right)\right) \\
& \left.\leqslant \bigwedge_{C \in L^{Z}} \mathbb{S}\left(f_{L}^{\leftarrow}\left(\mathcal{N}_{X}\left(g_{L}^{\leftarrow}(C)\right)\right), \mathcal{N}_{Y}\left(f_{L}^{\leftarrow}\left(g_{L}^{\leftarrow}(C)\right)\right)\right)\right) \\
& \wedge \bigwedge_{B \in L^{Z}} \mathbb{S}\left(g_{L}^{\leftarrow}\left(\mathcal{N}_{Z}(B)\right), \mathcal{N}_{Y}\left(g_{L}^{\leftarrow}(B)\right)\right) \\
& \left.\leqslant \bigwedge_{C \in L^{Z}} \mathbb{S}\left(f_{L}^{\leftarrow}\left(\mathcal{N}_{X}\left(g_{L}^{\leftarrow}(C)\right)\right), \mathcal{N}_{Y}\left(f_{L}^{\leftarrow}\left(g_{L}^{\leftarrow}(C)\right)\right)\right)\right) \\
& \wedge \bigwedge_{B \in L^{Z}} \mathbb{S}\left(f_{L}^{\leftarrow}\left(g_{L}^{\leftarrow}\left(\mathcal{N}_{Z}(B)\right)\right), f_{L}^{\leftarrow}\left(\mathcal{N}_{Y}\left(g_{L}^{\leftarrow}(B)\right)\right)\right) \\
& \left.\leqslant \bigwedge_{B \in L^{Z}} \mathbb{S}\left(f_{L}^{\leftarrow}\left(\mathcal{N}_{X}\left(g_{L}^{\leftarrow}(B)\right)\right), \mathcal{N}_{Y}\left(f_{L}^{\leftarrow}\left(g_{L}^{\leftarrow}(B)\right)\right)\right)\right)
\end{aligned}
$$

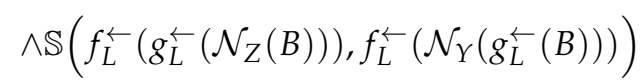

$$
\begin{aligned}
& \left.\leqslant \bigwedge_{B \in L^{Z}} \mathbb{S}\left(f_{L}^{\leftarrow}\left(g_{L}^{\leftarrow}\left(\mathcal{N}_{Z}(B)\right)\right), \mathcal{N}_{Y}\left(f_{L}^{\leftarrow}\left(g_{L}^{\leftarrow}(B)\right)\right)\right)\right) \\
& =\bigwedge_{B \in L^{Z}} \mathbb{S}\left((g \circ f)_{L}^{\overleftarrow{L}}\left(\mathcal{N}_{Z}(B)\right), \mathcal{N}_{X}\left((g \circ f)_{L}^{\overleftarrow{L}}(B)\right)\right) \\
& =D_{c_{2}}(g \circ f) \text {. }
\end{aligned}
$$

(2) Adopting the proof of (1), it can be verified directly.

Next, we investigate the connections between degrees of $L$-continuity and that of $L$-openness.

Theorem 12. For L-topological spaces $\left(X, \mathcal{T}_{X}\right),\left(Y, \mathcal{T}_{Y}\right)$ and $\left(Z, \mathcal{T}_{Z}\right)$, if $f$ is surjective, then the following inequality holds:

$$
D_{k}(g \circ f) \wedge D_{c_{2}}(f) \leqslant D_{k}(g) .
$$

Proof. Since $f$ is surjective, we have $f_{L} \rightarrow\left(f_{L}^{\leftarrow}(C)\right)=C$ for all $C \in L^{Y}$. Then, it follows that

$$
\begin{aligned}
& \bigwedge_{B \in L^{Y}} \mathbb{S}\left(g_{\vec{L}}\left(\mathcal{N}_{Y}(B)\right), \mathcal{N}_{Z}\left(g_{L}(B)\right)\right) \\
= & \bigwedge_{B \in L^{Y}} \mathbb{S}\left(g_{\vec{L}}\left(\mathcal{N}_{Y}\left(f_{L} \vec{L}\left(f_{L}^{\leftarrow}(B)\right)\right)\right), \mathcal{N}_{Z}\left(g_{L}\left(f_{L}\left(f_{L}^{\leftarrow}(B)\right)\right)\right)\right) \\
\geqslant & \bigwedge_{A \in L^{X}} \mathbb{S}\left(g_{\vec{L}}\left(\mathcal{N}_{Y}\left(f_{L}(A)\right)\right), \mathcal{N}_{Z}((g \circ f) \vec{L}(A))\right) \\
\geqslant & \bigwedge_{B \in L^{Y}} \mathbb{S}\left(g_{L} \vec{L}\left(\mathcal{N}_{Y}(B)\right), \mathcal{N}_{Z}\left(g_{L}(B)\right)\right) .
\end{aligned}
$$


This shows

$$
\bigwedge_{B \in L^{Y}} \mathbb{S}\left(g_{\vec{L}}\left(\mathcal{N}_{Y}(B)\right), \mathcal{N}_{Z}\left(g_{L} \vec{L}(B)\right)\right)=\bigwedge_{A \in L^{X}} \mathbb{S}\left(g_{L}\left(\mathcal{N}_{Y}\left(f_{L}(A)\right)\right), \mathcal{N}_{Z}\left((g \circ f)_{L}(A)\right)\right)
$$

Then, we have

$$
\begin{aligned}
& D_{k}(g \circ f) \wedge D_{c_{2}}(f) \\
= & \bigwedge_{A \in L^{X}} \mathbb{S}\left((g \circ f) \vec{L}\left(\mathcal{N}_{X}(A)\right), \mathcal{N}_{Z}((g \circ f) \vec{L}(A))\right) \\
& \wedge \bigwedge_{B \in L^{Y}} \mathbb{S}\left(f_{L}^{\leftarrow}\left(\mathcal{N}_{Y}(B)\right), \mathcal{N}_{X}\left(f_{L}^{\leftarrow}(B)\right)\right) \\
\leqslant & \bigwedge_{A \in L^{X}} \mathbb{S}\left((g \circ f)_{L}\left(\mathcal{N}_{X}(A)\right), \mathcal{N}_{Z}\left((g \circ f)_{L}(A)\right)\right) \\
& \left.\wedge \bigwedge_{A \in L^{X}} \mathbb{S}\left(g_{L} \overrightarrow{\mathcal{N}_{Y}}\left(f_{L}(A)\right)\right),(g \circ f)_{L}\left(\mathcal{N}_{X}(A)\right)\right) \\
\leqslant & \bigwedge_{A \in L^{X}} \mathbb{S}\left(g_{L}\left(\mathcal{N}_{Y}\left(f_{L}(A)\right)\right), \mathcal{N}_{Z}\left((g \circ f)_{L}(A)\right)\right) \\
= & \bigwedge_{B \in L^{Y}} \mathbb{S}\left(\mathcal{N}_{Z}\left(g_{L}\left(\mathcal{N}_{Y}(B)\right), g_{L}(B)\right)\right) \\
= & D_{k}(g) .
\end{aligned}
$$

Theorem 13. For L-topological spaces $\left(X, \mathcal{T}_{X}\right),\left(Y, \mathcal{T}_{Y}\right)$ and $\left(Z, \mathcal{T}_{Z}\right)$, if $f$ is injective, then the following inequality holds:

$$
D_{k}(g \circ f) \wedge D_{c_{2}}(g) \leqslant D_{k}(f) .
$$

Proof. Since $g$ is injective, we have $g_{L}^{\overleftarrow{L}}\left(g_{L}(C)\right)=C$ for all $C \in L^{Y}$. Then, it follows that

$$
\begin{aligned}
& D_{k}(g \circ f) \wedge D_{\mathcal{C}_{2}}(g) \\
& =\bigwedge_{B \in L^{X}} \mathbb{S}\left((g \circ f)_{L}\left(\mathcal{N}_{X}(B)\right), \mathcal{N}_{Z}\left((g \circ f)_{L}(B)\right)\right) \\
& \wedge \bigwedge_{C \in L^{Z}} \mathbb{S}\left(g_{L}^{\leftarrow}\left(\mathcal{N}_{Z}(C)\right), \mathcal{N}_{Y}\left(g_{L}^{\leftarrow}(C)\right)\right) \\
& \leqslant \bigwedge_{B \in L^{X}} \mathbb{S}\left(g_{\vec{L}}\left((g \circ f)_{L}\left(\mathcal{N}_{X}(B)\right)\right), g_{L} \rightarrow\left(\mathcal{N}_{Z}\left((g \circ f)_{L}(B)\right)\right)\right) \\
& \wedge \bigwedge_{B \in L^{X}} \mathbb{S}\left(g_{L}^{\leftarrow}\left(\mathcal{N}_{Z}\left((g \circ f)_{L}(B)\right)\right), \mathcal{N}_{Y}\left(g_{L}^{\leftarrow}\left((g \circ f)_{L}(B)\right)\right)\right) \\
& =\bigwedge_{B \in L^{X}} \mathbb{S}\left(f_{L} \rightarrow\left(\mathcal{N}_{X}(B), \mathcal{N}_{Y}\left(f_{L} \rightarrow(B)\right)\right)\right) \\
& =D_{k}(f) \text {. }
\end{aligned}
$$

Theorem 14. Suppose that $L$ is a frame with an order-reversing involution', where $a^{\prime}=a \rightarrow \perp$ for $a \in L$. For an L-topological space $(X, \mathcal{T}), \mathcal{C}=\left\{B \mid B^{\prime} \in \mathcal{T}\right\}$ is an L-cotopology and $C l(B)=\left(\mathcal{N}\left(B^{\prime}\right)\right)^{\prime}$. 
Proof. It is easy to verify that $\mathcal{C}$ is an $L$-cotopology and $\operatorname{Cl}(A)=\left(\mathcal{N}\left(A^{\prime}\right)\right)^{\prime}$.

Theorem 15. Suppose that $L$ is a frame with an order-reversing involution', where $a^{\prime}=a \rightarrow \perp$ for $a \in$ L. For L-topological spaces $\left(X, \mathcal{T}_{X}\right)$ and $\left(Y, \mathcal{T}_{Y}\right), D_{c_{1}}(f)=D_{c_{2}}(f)$.

Proof. By Theorems 5 and 14,

$$
\begin{aligned}
D_{c_{1}}(f) & =\bigwedge_{B \in L^{Y}} \mathbb{S}\left(C l_{X}\left(f_{L}^{\leftarrow}(B)\right), f_{L}^{\leftarrow}\left(C l_{Y}(B)\right)\right) \\
& =\bigwedge_{B \in L^{Y}} \mathbb{S}\left(\left(\mathcal{N}_{X}\left(f_{L}^{\leftarrow}\left(B^{\prime}\right)\right)\right)^{\prime}, f_{L}^{\leftarrow}\left(\left(\mathcal{N}_{Y}\left(B^{\prime}\right)\right)^{\prime}\right)\right) \\
& =\bigwedge_{B \in L^{Y}} \mathbb{S}\left(f_{L}^{\leftarrow}\left(\mathcal{N}_{Y}\left(B^{\prime}\right)\right), \mathcal{N}_{X}\left(f_{L}^{\leftarrow}\left(B^{\prime}\right)\right)\right) \\
& =\bigwedge_{B \in L^{Y}} \mathbb{S}\left(f_{L}^{\leftarrow}\left(\mathcal{N}_{Y}(B)\right), \mathcal{N}_{X}\left(f_{L}^{\leftarrow}(B)\right)\right) \\
& =D_{\mathcal{c}_{2}}(f) .
\end{aligned}
$$

\section{Conclusions}

In this paper, we equip each mapping between $L$-cotopological spaces with some degree to be an $L$-continuous mapping and an $L$-closed mapping, and equip each mapping between $L$-topological spaces with some degree to be an $L$-continuous mapping and an $L$-open mapping. From this aspect, we could consider the degrees of $L$-continuity, $L$-closedness and $L$-openness for a mapping even if the mapping is not a continuous mapping, a closed mapping or an open mapping. By means of these definitions, we proved that the degrees of $L$-continuity, $L$-closedness and $L$-openness for mappings naturally suggest lattice-valued logical extensions of properties related to continuous mappings, closed mappings and open mappings in classical topological spaces to fuzzy topological spaces. Moreover, if $L$ is a frame with an order-reversing involution ', where $b^{\prime}=b \rightarrow \perp$ for $b \in L$, then degrees of $L$-continuity for mappings between $L$-cotopological spaces and degrees of $L$-continuity for mappings between $L$-topological spaces are equivalent.

As future research, we will consider the following two problems:

(1) By means of the degree method, we can also define the degrees of some topological properties. For example, we can use the convergence degree of a fuzzy ultrafilter to define the compactness degree of an $L$-topological space.

(2) Based on the degrees of $L$-continuity, $L$-openness and $L$-closedness for mappings, we can further define the degrees of $L$-homeomorphism in a degree. We only need to equip a bijective mapping with the degrees of $L$-continuity and $L$-openness.

Author Contributions: Z.X. contributed the central idea. Z.X. and Q.L. writed this manuscript and revised it.

Funding: This work is supported by the National Natural Science Foundation of China $(11871097,11971448)$, the Project (2017M622563) funded by China Postdoctoral Science Foundation and the Project (KYTZ201631, CRF201611, 2017Z056) Supported by the Scientific Research Foundation of CUIT.

Acknowledgments: The authors thank the reviewers and the editor for their valuable comments and suggestions.

Conflicts of Interest: The authors declare no conflict of interest. 


\section{References}

1. Chang, C.L. Fuzzy topological spaces. J. Math. Anal. Appl. 1968, 24, 182-190. [CrossRef]

2. Fang, J.M. Stratified L-ordered quasiuniform limit spaces. Fuzzy Sets Syst. 2013, 227, 51-73. [CrossRef]

3. Jäger, G. A category of L-fuzzy convergence spaces. Quaest. Math. 2001, 24, 501-517. [CrossRef]

4. Jin, Q.; Li, L.Q.; Lv, Y.R.; Zhao, F.; Zou, J. Connectedness for lattice-valued subsets in lattice-valued convergence spaces. Quaest. Math. 2019, 42, 135-150. [CrossRef]

5. Kubiak, T. On Fuzzy Topologies. Ph.D.Thesis, Adam Mickiewicz, Poznan, Poland, 1985.

6. Lowen, E.; Lowen, R.; Wuyts, P. The categorical topological approach to fuzzy topology and fuzzy convergence. Fuzzy Sets Syst. 1991, 40, 347-373. [CrossRef]

7. Li, L.Q.; Jin, Q.; Hu, K. Lattice-valued convergence associated with CNS spaces. Fuzzy Sets Syst. 2019, 370, 91-98. [CrossRef]

8. Li, L.Q. p-Topologicalness-A Relative Topologicalness in T-Convergence Spaces. Mathematics 2019, 7, 228. [CrossRef]

9. Pang, B. Convergence structures in M-fuzzifying convex spaces. Quaest. Math. 2019. [CrossRef]

10. Pang, B.; Shi, F.G. Strong inclusion orders between $L$-subsets and its applications in $L$-convex spaces. Quaest. Math. 2018, 41, 1021-1043. [CrossRef]

11. Pang, B.; Shi, F.G. Fuzzy counterparts of hull operators and interval operators in the framework of L-convex spaces. Fuzzy Sets Syst. 2019, 369, 20-39. [CrossRef]

12. Pang, B.; Xiu, Z.Y. An axiomatic approach to bases and subbases in $L$-convex spaces and their applications. Fuzzy Sets Syst. 2019, 369, 40-56. [CrossRef]

13. Shi, F.G.; Xiu, Z.Y. ( L, M)-fuzzy convex structures. J. Nonlinear Sci. Appl. 2017, 10, 3655-3669. [CrossRef]

14. Sostak, A.P. On a fuzzy topological structure. Rend. Circ. Mat. Palermo (Suppl. Ser. II) 1985, 11, 89-103.

15. Wang, B.; Li, Q.; Xiu, Z.Y. A categorical approach to abstract convex spaces and interval spaces. Open Math. 2019, 17, 374-384. [CrossRef]

16. Wang, L.; Pang, B. Coreflectivities of $(L, M)$-fuzzy convex structures and $(L, M)$-fuzzy cotopologies in ( $L, M)$-fuzzy closure systems. J. Intell. Fuzzy Syst. 2019. [CrossRef]

17. Xiu, Z.Y.; Pang, B. M-fuzzifying cotopological spaces and $M$-fuzzifying convex spaces as $M$-fuzzifying closure spaces. J. Intell. Fuzzy Syst. 2017, 33, 613-620. [CrossRef]

18. Zhang, D.X. An enriched category to many valued topology. Fuzzy Sets Syst. 2007, 158, 349-466. [CrossRef]

19. Yue, Y.L.; Fang, J.M. On separation axioms in I-fuzzy topological spaces. Fuzzy Sets Syst. 2006, 157, 780-793. [CrossRef]

20. Shi, F.G. (L, M)-fuzzy metric spaces. Indian J. Math. 2010, 52, 1-20.

21. Shi, F.G. Regularity and normality of $(L, M)$-fuzzy topological spaces. Fuzzy Sets Syst. 2011, 182, 37-52. [CrossRef]

22. Li, H.Y.; Shi, F.G. Degrees of fuzzy compactness in L-fuzzy topological spaces. Fuzzy Sets Syst. 2010, 161, 988-1001. [CrossRef]

23. Pang, B.; Shi, F.G. Degrees of compactness of $(L, M)$-fuzzy convergence spaces and its applications. Fuzzy Sets Syst. 2014, 251, 1-22. [CrossRef]

24. Pang, B. Degrees of separation properties in stratified $L$-generalized convergence spaces using residual implication. Filomat 2017, 31, 6293-6305. [CrossRef]

25. Wang, L.; Wu, X.Y.; Xiu, Z.Y. A degree approach to relationship among fuzzy convex structures, fuzzy closure systems and fuzzy Alexandrov topologies. Open Math. 2019, 17, 913-928. [CrossRef]

26. Xiu, Z.Y.; Li, Q.G. Relations among $(L, M)$-fuzzy convex structures, $(L, M)$-fuzzy closure systems and ( $L, M)$-fuzzy Alexandrov topologies in a degree sense. J. Intell. Fuzzy Syst. 2019, 36, 385-396. [CrossRef]

27. Xiu, Z.Y.; Pang, B. A degree approach to special mappings between M-fuzzifying convex spaces. J. Intell. Fuzzy Syst. 2018, 35, 705-716. [CrossRef]

28. Pang, B. Degrees of continuous mappings, open mappings, and closed mappings in $L$-fuzzifying topological spaces. J. Intell. Fuzzy Syst. 2014, 27, 805-816. 
29. Liang, C.Y.; Shi, F.G.; Degree of continuity for mappings of $(L, M)$-fuzzy topological spaces. J. Intell. Fuzzy Syst. 2014, 27, 2665-2677.

30. Li, Q.H.; Huang, H.L.; Xiu, Z.-Y. Degrees of special mappings in the theory of L-convex spaces. J. Intell. Fuzzy Syst. 2019, 37, 2265-2274. [CrossRef]

31. Bělohlávek, R. Fuzzy Relational Systems, Foundations and Principles; Kluwer Academic/Plenum Publishers: New York, NY, USA; Boston, MA, USA; Dordrecht, The Netherlands; London, UK; Moscow, Russia, 2002.

32. Höhle, U.; Šostak, A.P. Axiomatic foudations of fixed-basis fuzzy topology. In Mathematics of Fuzzy Sets: Logic, Topology, and Measure Theory, Handbook Series; Höhle, U., Rodabaugh, S.E., Eds.; Kluwer Academic Publishers: Boston, MA, USA; Dordrecht, The Netherlands; London, UK, 1999; Volume 3, pp. 123-173. 33. Liu, Y.M.; Luo, M.K. Fuzzy Topology; World Scientific Publication: Singapore, 1998.

(C) 2019 by the authors. Licensee MDPI, Basel, Switzerland. This article is an open access article distributed under the terms and conditions of the Creative Commons Attribution (CC BY) license (http:/ / creativecommons.org/licenses/by/4.0/). 